\title{
Analytical Analysis of Nonlinear Internal Resonance Bandgap of Pendulum Type Metamaterial
}

SHAOJIE GUO ( $\square$ guoshaojie159@stu.xjtu.edu.cn )

Xi'an Jiaotong University https://orcid.org/0000-0001-6365-8684

\section{Changqing Bai}

Xi'an Jiaotong University

Hongyan Zhang

Chang'an University

Yahong Zhang

Xi'an Jiaotong University

\section{Research Article}

Keywords: Pendulum type metamaterial, nonlinear dynamic model, Dispersion characteristics, Theoretical model, Internal resonance, Nonlinear bandgap

Posted Date: October 25th, 2021

DOl: https://doi.org/10.21203/rs.3.rs-918811/v1

License: (c) (1) This work is licensed under a Creative Commons Attribution 4.0 International License. Read Full License 


\title{
Analytical Analysis of Nonlinear Internal Resonance Bandgap of Pendulum Type Metamaterial
}

\author{
Shaojie Guo ${ }^{1}$, Changqing Bai ${ }^{1}{ }^{*}$, Hongyan Zhang ${ }^{2}$, Yahong Zhang1 \\ ${ }^{1}$ State Key Laboratory for Strength and Vibration of Mechanical Structures / Shaanxi Key \\ Laboratory of Environment and Control for Flight Vehicle, Xi' an Jiaotong \\ University, Xi'an, 710049, China \\ ${ }^{2}$ School of Science, Chang' an University, Xi' an 710064, China \\ baichq@mail.xjtu.edu.cn
}

\section{Abstract}

In this paper, a pendulum type metamaterial (PTM) is designed with a pendulum bob hinged at the primary mass. The nonlinear dynamic model of PTM unit cell is presented with the aid of the Bloch theorem. The analytical formula of dispersion equation is deduced from the nonlinear model using the harmonic balance method. The obtained bandgap of the innovative metamaterial is in good agreement with the numerical result, thus validating the presented model. The nonlinear frequency band structure of PTM is investigated and compared with that obtained from linearized PTM model. It is found that the local resonant bandgap is tunable, and the nonlinear geometric influence of pendulum on PTM bandwidth is significant. The effects of $1: 1,1: 1 / 2$ and $1: 1 / 3$ internal resonance on the dispersion characteristics are analyzed with large swinging angle. The upper boundaries 
of the frequency bandgap under $1: 1 / 2$ and $1: 1 / 3$ internal resonance rise nonlinearly with the characteristic frequency of the secondary system to higher than those under linear and 1:1 internal resonance conditions. The mass coefficient gradually increases the width of PTM bandgap and PTM can possess a broader bandgap with optimized parameters.

Keywords: Pendulum type metamaterial; nonlinear dynamic model;

Dispersion characteristics; Theoretical model; Internal resonance;

Nonlinear bandgap

\section{Introduction}

The increasing popularity of functional materials has led to an increasing interest in the design and manufacture of the new materials for their unique properties. Metamaterial with manmade microstructure exhibits extraordinary behaviors such as negative effective mass density, negative refractive index and so on. Potential applications of these unconventional metamaterials, which do not exist in nature, include vibration isolation [1-3], acoustic wave control [4-8] and blast-wave mitigation [9-11]. The special and important feature of the metamaterials in vibration and wave attenuation is derived from the existence of the bandgaps, within which the transmission of the wave is prohibited.

In early researches, metamaterials with local resonant structure are explored to have bandgaps at lower frequency for vibration attenuation. After Liu [4] constructed a 
novel sonic metamaterial consisting of solid core material and coating of elastically soft material, more and more attention has been paid to the design of the elastic metamaterial with various local resonators such as mass-spring resonators [12], cantilever beam oscillators [13], Helmholtz resonators [5, 14], membrane with attached masses [15-16]. Recently, using a sub-wavelength array of beams attached to the plate, Lott [17] created a narrow transmission bands at the flexural resonances of the beams inside the wide frequency bandgap. A metamaterial terahertz biosensor, which has two resonant absorption frequencies, was represented by Li [18] for fast and label-free identification of early-stage cervical cancerous tissues. Seeking for the low-frequency band gap, metamaterials with different microstructure were designed and presented by Zhang [19] and Lin [20], respectively. In these works, it could be noted that the nonlinear factors were not taken into consideration. Therefore, some dynamic features related to the nonlinear conditions, such as the variation of natural frequencies, the hardening or softening behavior, the bistability, the internal resonance and so on, would not be discovered or discussed.

As can be seen from the current research progress, many scholars focus on seeking inspiration from the existing vibration or noise reduction methods and propose innovative metamaterial configuration for better vibration control. Using a mass-spring absorber with a softening and hardening spring, Roberson [21] has found that the nonlinear absorber offers a significant advantage over the corresponding linear absorber. In Ref. [22], in view of the classical linear vibration absorber, a nonlinear pendulum model was introduced by Yurchenko to addresses the nonlinear vibration mitigation 
strategies of a mechanical system. The bi-stability of nonlinear vibration absorber systems, which can result in the multiple stable equilibrium states and the snap-through behaviors, is investigated by Alevras [23], Nishi et al. [24], and Benacchio [25]. The internal resonance, as another important feature of the nonlinear systems, plays an important part in the nonlinear dynamic response of the vibration isolation devices. The autoparametric vibration absorber, whose good vibration reduction performance has been discovered and verified in practice, has attracted the attention of many researchers. Especially, the internal resonance of the pendulum type absorbers, whose oscillator is hinged by a simple pendulum, have attracted an increasing interest [26-28]. It was represented by Haxton [29] and Emrah [30] that one-to-two internal resonance of their proposed vibration absorber would benefit from the saturation phenomenon for vibration absorption. Besides, other types of resonance have also been examined such as the one-to-one internal resonance [31], the one-to-two-to-four internal resonance [32] and three-to-one internal resonance [33], the one-to-one-to zero internal resonance [34]. It is interesting to design a novel metamaterial based on the mechanism of autoparametric vibration absorber. Since the nonlinearity possesses an important impact on the property of these vibration absorber, the nonlinear effect would be also significant in the novel metamaterial.

In metamaterial analysis researches, more and more published papers had studied the nonlinear dynamic behavior of metamaterials as the nonlinear factors are taken into account. The approximate dispersion characteristics of the locally resonant metamaterial subject to weak nonlinearities were represented by Fronk [35], and Kevin 
[36]. Using geometric nonlinearities, Bilal [37] presented a realization of a transistorlike device, controlled by magnetic coupling, which can gate, switch, and cascade phonons without resorting to frequency conversion. The study, which proposed by Fang [38], showed that the metamaterial with enhanced nonlinearity have self-growing band gaps and an adaptive-broadening band. Moreover, to realize the broadband and highly efficient vibration reduction in linear and nonlinear regimes, metamaterials with different configurations were presented by Sheng [39] and Brooke [40]. Up to now, researches on the nonlinear effect of the dynamic behavior of metamaterials, especially the phenomenon of internal resonance, are still scarce, which results in the difficulties in theoretical analysis and application of nonlinear metamaterials.

To sum up, a pendulum type metamaterial (PTM) is designed and presented on the basis of the damping mechanism of the autoparametric vibration absorber in this paper. The harmonic balance method and the Bloch theorem are employed in obtaining the analytical dispersion equations of the novel metamaterial. It is intended to cast light on the frequency band structure of the nonlinear metamaterial using theoretical approach.

\section{Pendulum Type Metamaterial and Nonlinear Dynamic Modelling}

As shown in Fig. 1, a pendulum type metamaterial (PTM) is designed on the basis of the damping mechanism of the autoparametric vibration absorber. The novel metamaterial is composed of an array of spring-mass-pendulum resonator, where the resonators are connected to each other by springs. The pendulums, whose motion possesses strongly nonlinear characteristics under the large swinging angle, have an 
important influence on the dynamic behavior of PTM. Considering the large swinging angle, the motion of pendulum cannot be simplified as that of spring oscillator, which is obviously different from the traditional analysis approach. Thus, in order to obtain the nonlinear bandgap of PTM, we make an attempt to establish 2 DOF dynamic model of the presented metamaterial in the following section.

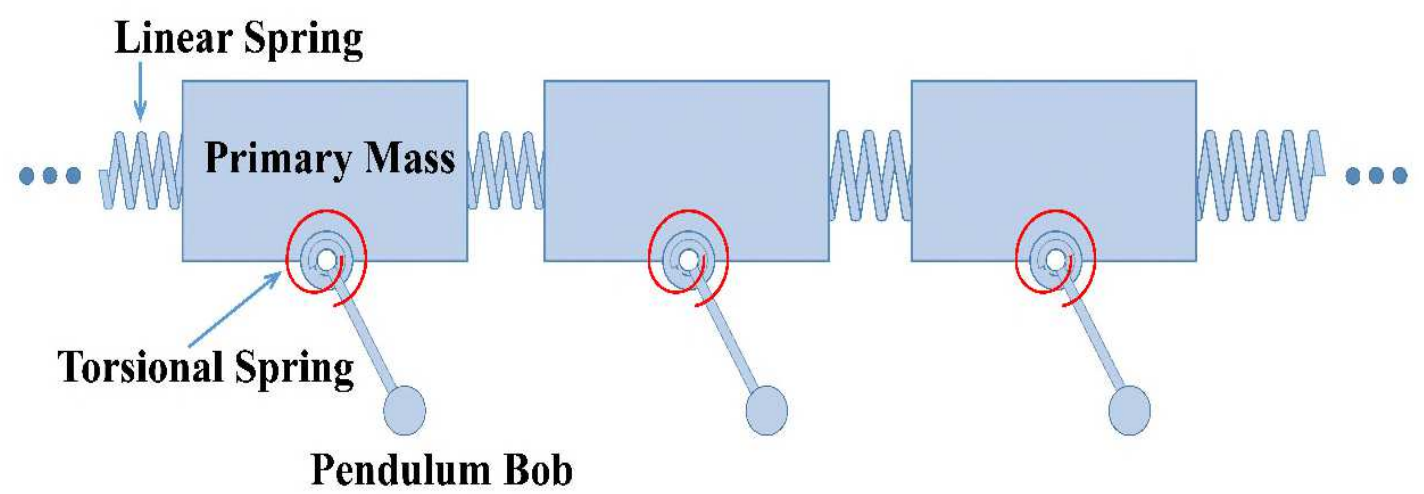

Fig. 1 The schematic drawing of PTM

Without loss of generality, we can consider the infinite periodic structure using representative unit cells with suitable periodic boundary conditions. As shown in Fig. 2, the unit cell of the proposed metamaterial consists of two parts: the primary system and the secondary system. The mass $M$ and the linear spring with stiffiness $K_{l}$ form the primary system. The secondary system comprises of a simple pendulum of length $l$ hinged at the primary mass $M$. The mass of the pendulum bob is denoted as $m$ and $K_{\theta}$ is the torsional stiffness of the spring resisting the angular motion of the pendulum. The coordinate system in Fig. 2 is defined as $O X Y$, where $X, Y$ are the horizontal and vertical coordinate directions and the origin $O$ is fixed to the center of the primary mass $M$. Using this coordinate system, the kinetic energy of the unit cell is obtained as:

$$
T=\frac{1}{2}(M+m) \cdot z^{2}+\frac{1}{2} m l^{2} \theta^{2}+m l x \cos \theta
$$


where $x$ is the displacement of $M, \theta$ is the angle of rotation of the pendulum, and the dot denotes the derivative with respect to the time $t$. \& and $\theta^{\&}$ are the horizontal velocity of the primary mass and the angular velocity of the pendulum bob, respectively. The potential energy due to the elongation of the linear spring and the work done by gravity can also be expressed as

$$
U=\frac{1}{2} K_{1} x^{2}+\frac{1}{2} K_{\theta} \theta^{2}+m g l(1-\cos \theta)
$$

in which $g$ is the acceleration of gravity.

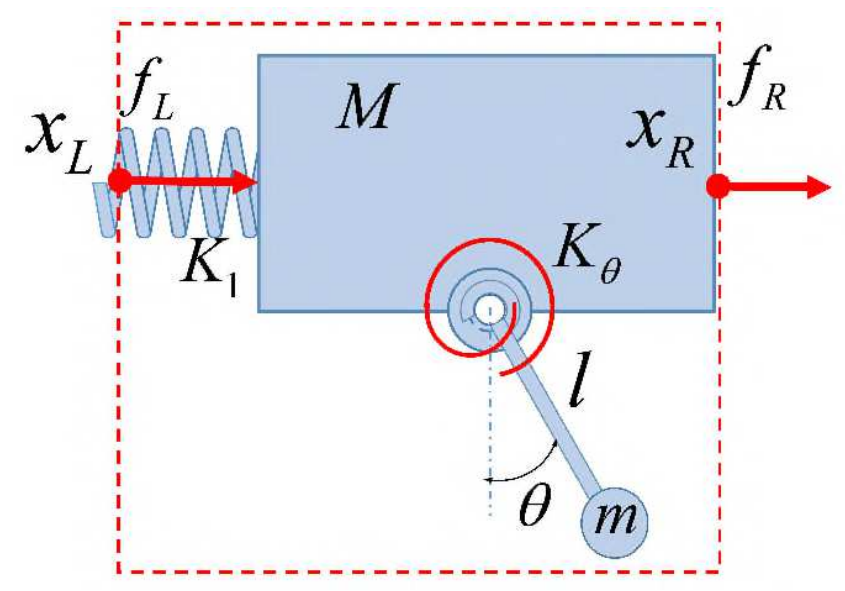

Fig. 2 The unit cell of the PTM

To obtain equation of motion of PTM, the following Lagrange's equation is employed

$$
\frac{d}{d t}\left(\frac{\partial T}{\partial \&}\right)-\frac{\partial T}{\partial q_{i}}+\frac{\partial U}{\partial q_{i}}=Q_{i}, \quad i=1,2 \mathrm{~L}
$$

where $q_{i}$ is the relatively generalized coordinate and $Q_{i}$ is the forces corresponding to generalized coordinates, including external loads and damping force. When there is not external force applying on the unit cell, the Lagrange's equation can be written as

$$
\left\{\begin{array}{l}
\frac{d}{d t}\left(\frac{\partial T}{\partial \&}\right)-\frac{\partial T}{\partial x}+\frac{\partial U}{\partial x}=0 \\
\frac{d}{d t}\left(\frac{\partial T}{\partial \theta^{\&}}\right)-\frac{\partial T}{\partial \theta}+\frac{\partial U}{\partial \theta}=0
\end{array}\right.
$$


Then, the expressions given in Eqs. (1) and (2) for $T$ and $U$ are introduced into Eqs.(4) and the nonlinear equations of motion of the unit cell can be obtained as

$$
\begin{aligned}
& (M+m) K_{1} x+m l\left(\operatorname{os} \theta-\theta^{2} \sin \theta\right)=0 \\
& m l^{2} K_{\theta} \theta+m l(\cos \theta+g \sin \theta)=0
\end{aligned}
$$

where and are the acceleration of mass and the angular acceleration of pendulum bob, respectively. When the primary mass is excited by an external harmonic load, Eq.(5) can be transformed to

$$
\begin{aligned}
& (M+m) K_{1} x+m l(\cos \theta-\sin \theta)=P_{0} \cos \omega t \\
& m l^{2} K_{\theta} \theta+m l(\cos \theta+g \sin \theta)=0
\end{aligned}
$$

where $P_{0}$ and $\omega$ are the amplitude and the angular velocity of the harmonic load.

The periodic boundary condition is applied on the unit cell in order to simulate the proposed infinite structure. In Fig. $2, f_{L}$ and $f_{R}$ are the force exerted by the adjacent unit cells on the left and the right, and the displacement of left end of the spring and the right end of the mass block are defined $x_{L}$ and $x_{R}$, respectively. It is noted that $x_{L}$ is equal to the displacement of the primary mass of the left unit cell. According to the Bloch theorem of periodic structure, the relationship between the left and right ends of the unit cell is given as

$$
\left\{\begin{array}{l}
x_{R}=x_{L} e^{-i q a} \\
f_{R}=-f_{L} e^{-i q a}
\end{array}\right.
$$

where $q$ and $a$ are the wave vector and the distance from the left end to the right end of the unit cell, respectively. Then, introducing Eq. (7) into Eq. (5), the nonlinear equations of motion of the unit cell can be finally written as 


$$
\begin{aligned}
& (M+m)+m l(\operatorname{os} \theta-\sin \theta)+K_{1} x_{L}[2-2 \cos (q a)]=0 \\
& m l^{2} K_{\theta} \theta+m l(\cos \theta+g \sin \theta)=0
\end{aligned}
$$

Here, we define the dimensionless parameters as follows:

$$
\begin{aligned}
& \psi=\frac{x_{L}}{l}, \quad \tau=\omega t, \quad r=\frac{m}{(M+m)} \\
& \omega_{1}=\sqrt{\frac{K_{1}}{(M+m)}}, \quad \omega_{2}=\sqrt{\frac{K_{\theta}}{m l^{2}}}, \quad \omega_{3}=\sqrt{\frac{g}{l}} \\
& \omega_{1}^{*}=\frac{\omega_{1}}{\omega}, \quad \omega_{2}{ }^{*}=\frac{\omega_{2}}{\omega}, \quad \omega_{3}^{*}=\frac{\omega_{3}}{\omega} \\
& \mu=\frac{\omega_{2}}{\omega_{1}}, \quad v=\frac{\omega_{3}}{\omega_{1}}
\end{aligned}
$$

Substituting Eqs. (9) into Eq. (8) and calculating the derivatives with respect to the dimensionless variables, $\omega_{1}{ }^{*}, \omega_{2}{ }^{*}$ and $\omega_{3}{ }^{*}$, one can obtained the nonlinear equations of motion of the unit cell in the following non-dimensional forms:

$$
\begin{aligned}
& \psi^{\prime \prime}+r \theta^{\prime \prime} \cos \theta-r \theta^{\prime 2} \sin \theta+\omega_{1}^{* 2} \psi[2-2 \cos (q a)]=0 \\
& \theta^{\prime \prime}+\omega_{2}{ }^{* 2} \theta+\psi^{\prime \prime} \cos \theta+\omega_{3}^{* 2} \sin \theta=0
\end{aligned}
$$

where the prime is denoted as the derivative with respect to the non-dimensional time, $\tau$.

\section{The nonlinear dispersion equation of PTM}

Equations (10) is in the couple nonlinear form, which might be difficult to be solved directly. In order to find the dispersion equation of the proposed PTM, the method of harmonic balance is employed in our research. Since the excitation is harmonic, the state solution of the primary mass could be also assumed to be harmonic with the same frequency. And the solution of Eq. (10) are assumed to be in the form

$$
\psi=\mathrm{A}_{1} \cos \left(\Omega_{1} \tau+\phi_{1}\right), \quad \theta=\mathrm{A}_{2} \cos \left(\Omega_{2} \tau+\phi_{2}\right)
$$


where A, $\Omega$ and $\phi$ are the amplitude of the dynamic response, the coefficient of the angular velocity and the initial phase, respectively. And the subscript " 1 " and " 2 " denote the accordingly parameters related to the primary mass and pendulum bob. Under the excitation, the motion of pendulum bob could resonate with a frequency near that of the primary mass. Considering the main internal resonance conditions, $\Omega_{2}$ can be taken as $1,1 / 2$ and $1 / 3$ when $\Omega_{1}$ is equal to 1 . And with respect to the solution of the presented dynamic model, the analysis of the nonlinear bandgap of PTM is carried out in the following sections.

\subsection{Case 1: $\Omega_{1}=1, \Omega_{2}=1$}

In this case, the period of the motion of the pendulum bob is equal to that of the primary mass. And we can derive the nonlinear bandgap from the proposed metamaterial, where PTM is under 1:1 internal resonance condition. To seek the bandgap of the presented PTM, Eq. (11) can be written as

$$
\psi=\mathrm{A}_{1} \cos \left(\tau+\phi_{1}\right), \quad \theta=\mathrm{A}_{2} \cos \left(\tau+\phi_{2}\right)
$$

Inserting Eq. (12) into Eq. (9), and the equation of motion can be changed to Eqs.

(13) and (14) as follows

$$
\begin{aligned}
& 2 \omega_{1}^{* 2} \mathrm{~A}_{1}[1-\cos (q a)] \cos \left(\tau+\phi_{1}\right)-\mathrm{A}_{1} \cos \left(\tau+\phi_{1}\right)-\mathrm{A}_{2} r \cos \left(\tau+\phi_{2}\right) \cos \left(\mathrm{A}_{2} \cos \left(\tau+\phi_{2}\right)\right) \\
& -r \mathrm{~A}_{2}^{2}\left(\frac{1-\cos \left(2 \tau+2 \phi_{2}\right)}{2}\right) \sin \left(\mathrm{A}_{2} \cos \left(\tau+\phi_{2}\right)\right)=0 \\
& -\mathrm{A}_{2} \cos \left(\tau+\phi_{2}\right)+\omega_{2}^{* 2} \mathrm{~A}_{2} \cos \left(\tau+\phi_{2}\right)-\mathrm{A}_{1} \cos \left(\tau+\phi_{1}\right) \cos \left(\mathrm{A}_{2} \cos \left(\tau+\phi_{2}\right)\right) \\
& +\omega_{3}^{* 2} \sin \left(\mathrm{A}_{2} \cos \left(\tau+\phi_{2}\right)\right)=0
\end{aligned}
$$

In order to solve these equations, the following Bessel's function is adopted 


$$
\begin{aligned}
& \sin \left(\mathrm{A}_{2} \cos \left(\tau+\phi_{2}\right)\right)=-2 \sum_{m=1}^{\infty}(-1)^{m} J_{2 m-1}\left(\mathrm{~A}_{2}\right) \cos \left[(2 m-1)\left(\tau+\phi_{2}\right)\right] \\
& \cos \left(\mathrm{A}_{2} \cos \left(\tau+\phi_{2}\right)\right)=J_{0}\left(\mathrm{~A}_{2}\right)+2 \sum_{m=1}^{\infty}(-1)^{m} J_{2 m}\left(\mathrm{~A}_{2}\right) \cos \left[(2 m)\left(\tau+\phi_{2}\right)\right]
\end{aligned}
$$

where $J_{2 m-1}\left(\mathrm{~A}_{2}\right)$ and $J_{2 m}\left(\mathrm{~A}_{2}\right)$ are the Bessel's coefficients.

The expression of $A_{l}$ can be obtained by introducing Eq. (15) into Eq. (13):

$$
\mathrm{A}_{1}=\frac{\left(\mathrm{A}_{2} r+\frac{r \mathrm{~A}_{2}^{3}}{8}+\frac{1}{96} r \mathrm{~A}_{2}^{5}\right) \cos \left(\tau+\phi_{2}\right)-\left(\frac{3}{8} r \mathrm{~A}_{2}^{3}+\frac{7}{384} r \mathrm{~A}_{2}{ }^{5}\right) \cos \left(3 \tau+3 \phi_{2}\right)+\frac{5}{384} r \mathrm{~A}_{2}{ }^{5} \cos \left(5 \tau+5 \phi_{2}\right)}{\left[2 \omega_{1}^{* 2}(1-\cos (q a))-1\right] \cos \left(\tau+\phi_{1}\right)}
$$

By substituting Eqs. (15) and (16) into Eq. (14), one obtains

$$
\begin{aligned}
& \left(+0.5\left(\omega_{3}^{* 2}-1+\omega_{2}^{* 2}\right)\left(2 \omega_{1}^{* 2}(1-\cos (q a))-1\right) \mathrm{A}_{2}-0.5 \mathrm{~A}_{2} r-\frac{1}{48} r \mathrm{~A}_{2}{ }^{5}+\frac{2}{294912} r \mathrm{~A}_{2}{ }^{9}\right) \cos \left(+\phi_{1}-\phi_{2}\right) \\
& +\left(0.5\left(\omega_{3}^{*_{2}}-1+\omega_{2}^{* 2}\right)\left(2 \omega_{1}^{* 2}(1-\cos (q a))-1\right) \mathrm{A}_{2}-0.5 \mathrm{~A}_{2} r-\frac{1}{48} r \mathrm{~A}_{2}{ }^{5}+\frac{2}{294912} r \mathrm{~A}_{2}{ }^{9}\right) \cos \left(2 \tau+\phi_{1}+\phi_{2}\right) \\
& +\left(+\frac{1}{4} r \mathrm{~A}_{2}^{3}-\frac{\omega_{3}^{* 2}}{48}\left(2 \omega_{1}^{* 2}(1-\cos (q a))-1\right) \mathrm{A}_{2}{ }^{3}+\frac{1}{64} r \mathrm{~A}_{2}{ }^{5}+\frac{1}{768} r \mathrm{~A}_{2}{ }^{7}-\frac{1}{73728} r \mathrm{~A}_{2}{ }^{9}\right) \cos \left(2 \tau-\phi_{1}+3 \phi_{2}\right) \\
& +\left(-\frac{\omega_{3}^{* 2}}{48}\left[2 \omega_{1}^{* 2}(1-\cos (q a))-1\right] \mathrm{A}_{2}{ }^{3}+\frac{1}{4} \mathrm{~A}_{2}{ }^{3} r+\frac{r \mathrm{~A}_{2}{ }^{5}}{64}+\frac{1}{768} r \mathrm{~A}_{2}{ }^{7}-\frac{1}{73728} r \mathrm{~A}_{2}{ }^{9}\right) \cos \left(4 \tau+\phi_{1}+3 \phi_{2}\right) \\
& -\left(\frac{1}{32} r \mathrm{~A}_{2}{ }^{5}+\frac{1}{768} r \mathrm{~A}_{2}{ }^{7}+\frac{1}{73728} r \mathrm{~A}_{2}{ }^{9}\right) \cos \left(4 \tau-\phi_{1}+5 \phi_{2}\right)-\left(\frac{1}{32} r \mathrm{~A}_{2}{ }^{5}+\frac{1}{768} r \mathrm{~A}_{2}{ }^{7}+\frac{1}{73728} r \mathrm{~A}_{2}{ }^{9}\right) \cos \left(6 \tau+\phi_{1}+5 \phi_{2}\right) \\
& +\left(\frac{1}{768} r \mathrm{~A}_{2}{ }^{7}+\frac{7}{294912} r \mathrm{~A}_{2}{ }^{9}\right) \cos \left(6 \tau-\phi_{1}+7 \phi_{2}\right)+\left(\frac{8}{6144} r \mathrm{~A}_{2}{ }^{7}+\frac{7}{294912} r \mathrm{~A}_{2}{ }^{9}\right) \cos \left(8 \tau+\phi_{1}+7 \phi_{2}\right) \\
& -\frac{5}{294912} r \mathrm{~A}_{2}{ }^{9} \cos \left(8 \tau-\phi_{1}+9 \phi_{2}\right)-\frac{5}{294912} r \mathrm{~A}_{2}{ }^{9} \cos \left(10 \tau+\phi_{1}+9 \phi_{2}\right)=0
\end{aligned}
$$

It can be found that Eq. (17) is a function of non-dimensional time, $\tau$, which is the sum of different harmonic terms. Equating the coefficients of the sine and cosine term, the following non-dimensional dispersion equation of PTM is obtained as

$$
\frac{1}{\omega_{1}^{*}}=\sqrt{\frac{2\left(v^{2}+\mu^{2}\right)(1-\cos (q a))}{\left(\begin{array}{l}
(1-\cos (q a)) \\
+0.5\left(v^{2}+\mu^{2}\right)
\end{array}\right) \sqrt{\left(\begin{array}{l}
\left.(1-\cos (q a))+0.5\left(v^{2}+\mu^{2}\right)\right)^{2} \\
-4\left(v^{2}+\mu^{2}\right)(1-\cos (q a))\left(\frac{1}{2}-\frac{1}{2} r-\frac{1}{48} r \mathrm{~A}_{2}{ }^{4}+\frac{1}{147456} r \mathrm{~A}_{2}^{8}\right.
\end{array}\right)}}}
$$

where the nonlinear bandgap of the proposed metamaterial is closely related to the mass coefficient $(r)$ and the dimensionless resonance frequency ( $\mu$ and $v$ ). 
3.2 Case 2: $\Omega_{l}=1, \Omega_{2}=1 / 2$

When the vibration of the pendulum bob has half the frequency of the primary mass, PTM is under 1:1/2 internal resonance condition. And the solution of Eq. (10) is given as

$$
\psi=\mathrm{A}_{1} \cos \left(\tau+\phi_{1}\right), \quad \theta=\mathrm{A}_{2} \cos \left(\frac{1}{2} \tau+\phi_{2}\right)
$$

Following the calculation process in 3.1 Section, we can obtain

$$
\begin{aligned}
& +\left(\begin{array}{l}
0.5\left(\omega_{2}^{* 2}-\frac{1}{4}+\omega_{3}^{* 2}\right)\left(2 \omega_{1}^{* 2}(1-\cos (q a))-1\right) \\
-\frac{1}{8} r-\frac{1}{192} r \mathrm{~A}_{2}{ }^{4}+\frac{1}{589824} r \mathrm{~A}_{2}^{8}
\end{array}\right) \cos \left(\frac{1}{2} \tau+\phi_{1}-\phi_{2}\right)+\left(\begin{array}{l}
\frac{1}{16} r \mathrm{~A}_{2}{ }^{2}-\frac{1}{48} \mathrm{~A}_{2}{ }^{2} \omega_{3}^{2}\left(2 \omega_{1}^{* 2}(1-\cos (q a))-1\right) \\
+\frac{1}{256} r \mathrm{~A}_{2}^{4}+\frac{1}{3072} r \mathrm{~A}_{2}{ }^{6}-\frac{1}{294912} r \mathrm{~A}_{2}^{8}
\end{array}\right) \cos \left(\frac{1}{2} \tau-\phi_{1}+3 \phi_{2}\right) \\
& +\left(\begin{array}{l}
0.5\left(\omega_{2}^{* 2}-\frac{1}{4}+\omega_{3}^{* 2}\right)\left(2 \omega_{1}^{* 2}(1-\cos (q a))-1\right) \\
-\frac{1}{8} r-\frac{1}{192} r \mathrm{~A}_{2}^{4}+\frac{1}{589824} r \mathrm{~A}_{2}^{8}
\end{array}\right) \cos \left(\frac{3}{2} \tau+\phi_{1}+\phi_{2}\right)+\left(-\frac{1}{128} r \mathrm{~A}_{2}{ }^{4}-\frac{1}{3072} r \mathrm{~A}_{2}{ }^{6}-\frac{1}{294912} r \mathrm{~A}_{2}^{8}\right) \cos \left(\frac{3}{2} \tau-\phi_{1}+5 \phi_{2}\right) \\
& +\left(\begin{array}{l}
-\frac{1}{48} \mathrm{~A}_{2}^{3} \omega_{3}^{{ }^{2}}\left(2 \omega_{1}^{\omega_{2}}(1-\cos (q a))-1\right)+\frac{1}{16} r \mathrm{~A}_{2}^{3} \\
+\frac{1}{256} r \mathrm{~A}_{2}^{5}+\frac{1}{3072} r \mathrm{~A}_{2}^{7}-\frac{1}{294912} r \mathrm{~A}_{2}{ }^{9}
\end{array}\right) \cos \left(\frac{5}{2} \tau+\phi_{1}+3 \phi_{2}\right)+\left(\frac{1}{3072} r \mathrm{~A}_{2}{ }^{7}+\frac{7}{1179648} r \mathrm{~A}_{2}^{9}\right) \cos \left(\frac{5}{2} \tau-\phi_{1}+7 \phi_{2}\right) \\
& +\left(-\frac{1}{128} r \mathrm{~A}_{2}^{5}-\frac{1}{3072} r \mathrm{~A}_{2}{ }^{7}-\frac{1}{294912} r \mathrm{~A}_{2}^{9}\right) \cos \left(\frac{7}{2} \tau+\phi_{1}+5 \phi_{2}\right)-\frac{5}{1179648} r \mathrm{~A}_{2}^{9} \cos \left(\frac{7}{2} \tau-\phi_{1}+9 \phi_{2}\right) \\
& +\left(\frac{1}{3072} r \mathrm{~A}_{2}^{7}+\frac{7}{1179648} r \mathrm{~A}_{2}^{9}\right) \cos \left(\frac{9}{2} \tau+\phi_{1}+7 \phi_{2}\right)-\frac{5}{1179648} r \mathrm{~A}_{2}^{9} \cos \left(\frac{11}{2} \tau+\phi_{1}+9 \phi_{2}\right)=0
\end{aligned}
$$

Balancing the different harmonic terms, the dimensionless dispersion equation of PTM under 1:1/2 internal resonance condition can be finally obtained as

$$
\frac{1}{\omega_{1}^{*}}=\frac{2\left(\left(\mu^{2}+v^{2}\right)(1-\cos (q a)) \cos \left(+\phi_{1}-\phi_{2}\right)-\frac{1}{24} \mathrm{~A}_{2}^{2}(1-\cos (q a)) v^{2} \cos \left(-\phi_{1}+3 \phi_{2}\right)\right)}{\left(\begin{array}{l}
\left(\frac{1}{48} \mathrm{~A}_{2}^{2} v^{2} \cos \left(-\phi_{1}+3 \phi_{2}\right)-\left(\frac{1}{4}(1-\cos (q a)) \alpha^{2}+0.5\left(\mu^{2}+v^{2}\right)\right) \cos \left(+\phi_{1}-\phi_{2}\right)\right)^{2} \cos \left(-\phi_{1}+3 \phi_{2}\right) \\
-\left(\begin{array}{l}
\frac{1}{4}(1-\cos (q a)) \alpha^{2} \\
+0.5\left(\mu^{2}+v^{2}\right)
\end{array}\right) \cos \left(+\phi_{1}-\phi_{2}\right)
\end{array}\right)}
$$


3.3 Case 3: $\Omega_{l}=1, \Omega_{2}=1 / 3$

When the presented metamaterial is under 1:1/3 internal resonance condition, the solution of the equations of motion of the unit cell can be written as

$$
\psi=\mathrm{A}_{1} \cos \left(\tau+\phi_{1}\right), \quad \theta=\mathrm{A}_{2} \cos \left(\frac{1}{3} \tau+\phi_{2}\right)
$$

The calculation procedure is similar to that above, and the non-dimensional dispersion equation of PTM is obtained as

$$
\frac{1}{\omega_{1}^{*}}=\sqrt{\left(\begin{array}{l}
\left.\left(\begin{array}{l}
0.5\left(\mu^{2}+v^{2}\right) \\
+\frac{1}{9}(1-\cos (q a))
\end{array}\right) \cos \left(+\phi_{1}-\phi_{2}\right)\right)^{2} \\
-\left(\begin{array}{l}
0.5\left(\mu^{2}+v^{2}\right) \\
+\frac{1}{9}(1-\cos (q a))
\end{array}\right) \cos \left(+\phi_{1}-\phi_{2}\right) \pm\left(\begin{array}{l}
\left.\left(-\mu^{2}-v^{2}\right)(1-\cos (q a)) \cos \left(+\phi_{1}-\phi_{2}\right)\right) \\
-4\left(\left(-\mu^{2}-v^{2}\right)(1-\cos (q a)) \cos \left(+\phi_{1}-\phi_{2}\right)\right)
\end{array}\left(\begin{array}{l}
-\frac{1}{18}+\frac{1}{18} r+\frac{1}{432} \mathrm{~A}_{2}{ }^{4} r \\
-\frac{1}{1327104} \mathrm{~A}_{2}^{8} r
\end{array}\right) \cos \left(+\phi_{1}-\phi_{2}\right)\right. \\
\left(\begin{array}{l}
\left.+\frac{1}{288} \mathrm{~A}_{2}{ }^{4}+\frac{1}{6912} \mathrm{~A}_{2}{ }^{6}\right) \\
+\frac{1}{663552} \mathrm{~A}_{2}^{8}{ }^{8}
\end{array}\right)
\end{array}\right.}
$$

\section{Nonlinear Bandgap Analysis of PTM}

According to the analytical dispersion equations shown in Eqs. (18), (21) and (23), the nonlinear bandgap of PTM can be analyzed and obtained. With respect to the solution of the presented dynamic model, the nonlinear bandgap analysis of PTM under various internal resonance conditions is carried out. The variation of the bandgap width of PTM versus non-dimensional parameter $(\mu, v$ and $r)$ is displayed in the following 
sections.

\subsection{The nonlinear bandgap of PTM under various internal resonance conditions}

Figure 3 illustrates the non-dimensional dispersion curves under various internal resonance conditions, where $q a$ and $1 / \omega_{1}{ }^{*}$ are the wave vector and the non-dimensional frequency, respectively. The non-dimensional parameters are given as $\mu=1.2669$, $v=0.0024$ and $r=0.4906$. The dimensionless dispersion curves are then compared to the results obtained from the linearized PTM dynamic model. The detail procedure of the linear modelling and bandgap calculation of PTM are given in Appendix A, where it is noted that the nonlinear terms in Eq. (8) are neglected. As shown in Fig. 3, it can be found that the bandgaps under different cases are significantly different from each other, in which each bandgap has a pair of upper and lower boundaries. Note that the lower boundaries of different cases are closely related to the characteristic frequency of the primary system of the unit cell, and the secondary system has a strong effect on the upper boundaries. Among them, the bandgap of PTM under 1:1 internal resonance condition (the black line) is similar to that obtained from the linear dynamic model (the red line), but the upper boundary of the bandgap of PTM under 1:1 internal resonance condition (the black line) is slightly higher than that of the linearized model, which is caused by the geometric nonlinearities of the secondary system. The lower boundaries of the bandgaps under $1: 1 / 2$ (blue line) and 1:1/3 (green line) internal resonances conditions vary from those under linear and 1:1 internal resonance conditions, which is due to the coupling effect of the torsion spring and the pendulum motion. The upper boundaries of the bandgaps caused by $1: 1 / 2$ and $1: 1 / 3$ internal resonances are much 
higher than those under linear and 1:1 internal resonance conditions. It is indicated that the nonlinear effect of secondary system of PTM could enhance the width of the bandgap.

For further analysis, the numerical method are employed to acquire the dynamic response under a specific harmonic excitation. As shown in Fig. 4, the frequency spectrum of PTM under the harmonic excitation versus the wave vector are illustrated and compared with theoretical results. It can be found from Fig. 4 (a), (b) and (c) that the boundaries of the bandgaps of PTM under different conditions are in a good agreement with the corresponding peak values in the frequency spectrum of the dynamic response (pink line), thus validating the presented PTM dynamic model. In Fig. 4 (a) and (b), when the non-dimensional frequency of the harmonic excitation is low, the frequency component of the characteristics frequency of the primary system has the highest magnitude. The values of the frequency components are equal to those of the lower boundaries of the bandgap under linear and 1:1 internal resonance conditions. We can see from Fig. 4 (c) that the four type of the boundary lines overlap together in the low-frequency areas, which is distinct from those in Fig. 4(a) and (b) markedly. The reason for this phenomenon is that the values of all the four lower boundaries become 0 as $q a$ gets closed to 0 . It can be concluded that the nonlinear effect has an important effect on the bandgap of the proposed PTM, which should be considered in the analysis. 


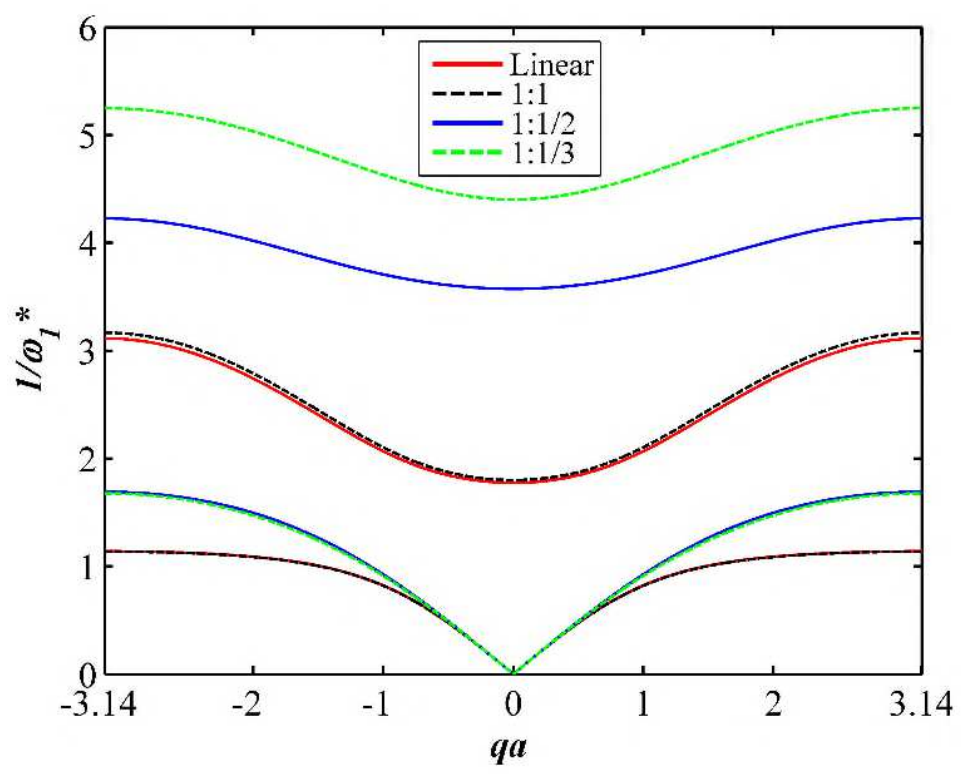

Fig. 3 The dimensionless dispersion curves of PTM under different cases

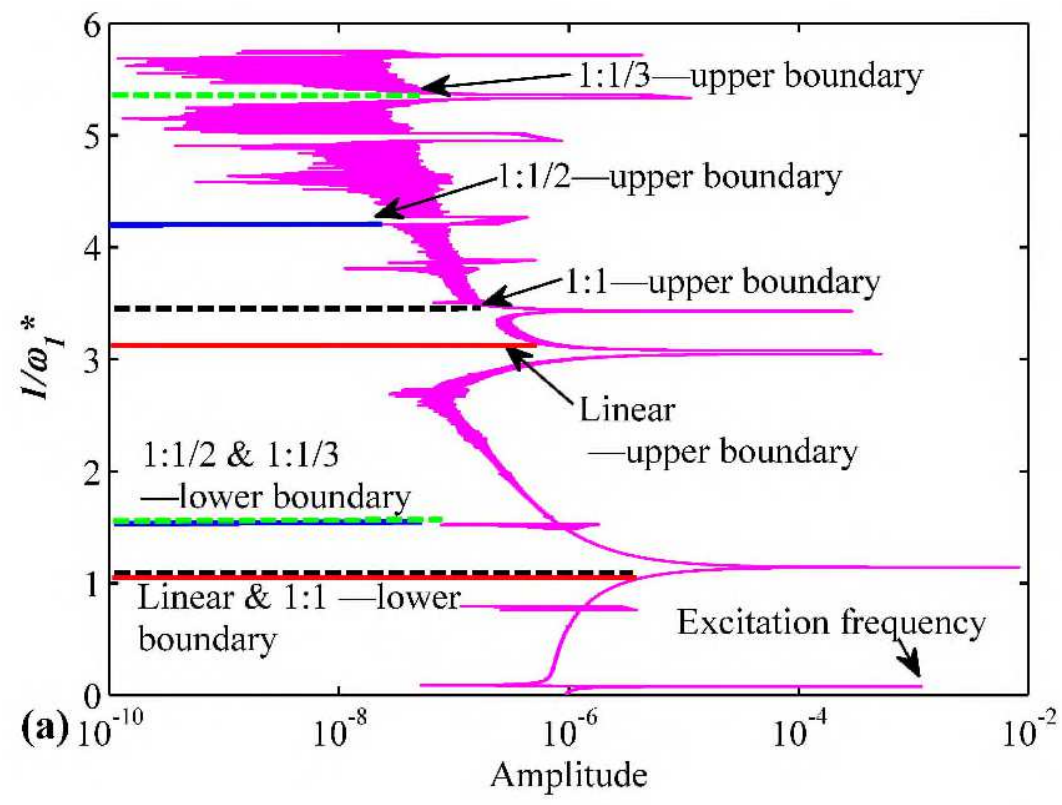



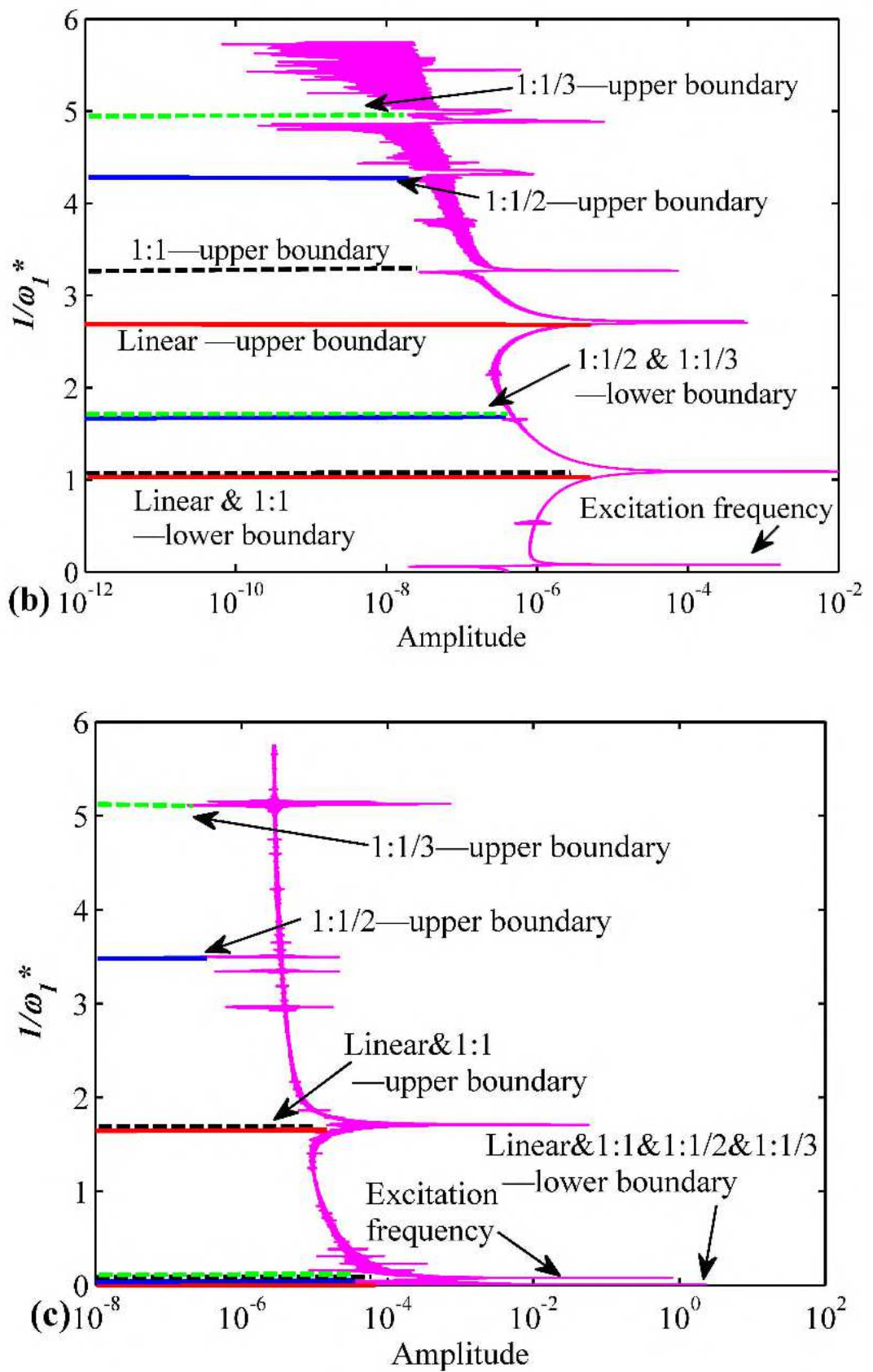

Fig. 4 The frequency spectrum of PTM under the harmonic excitation:

(a) $q a=-3.14$; (b) $q a=-2$; (c) $q a=0$.

\subsection{The nonlinear bandgap of PTM under various internal resonance conditions versus $\mu$}

According to the presented dynamic model, the nonlinear bandgap of PTM versus the non-dimensional parameter $\mu$ is investigated. The variation of the upper and lower 
boundaries of the bandgap with the increasing $\mu$ are represented in Fig. 5, where the vertical distances between the upper and the lower boundaries are the width of the bandgap at specific $\mu$. We can find from Fig. 5 that the amplitudes of the lower and upper boundaries under all the four conditions increase nonlinearly and differently with the growth of $\mu$. It is noted that the small value of $\mu$ has a slight influence on the upper boundaries of the bandgaps, where the total amplitude increases of the upper boundaries (blue lines) are not significant by comparing with other amplitude curves. However, as $\mu$ increases, the increase rate of the upper boundaries rise markedly faster than those of the lower boundaries (red lines). And then the distance between the two boundaries increases, which results in the growing width of the bandgap obtained from the linearized model and proposed dynamic model of PTM.

The dimensionless dispersion curves of PTM are plotted in Fig. 6(a-c), where $\mu$ is taken as $0.05,0.65$ and 3, respectively. From Fig. 6(a), it could be found that due to the effect of the secondary system the amplitudes of lower boundaries under $1: 1 / 2$ and $1: 1 / 3$ resonance conditions are twice and three times of those under linear and 1:1 internal conditions. However, as $\mu$ increases, the nonlinear impact of the pendulum bob motion on the primary mass decreases constantly, which causes the phenomenon of multiple frequency to become insignificant in Fig. 6(b-c). Moreover, the upper boundaries, which denote the characteristics frequency of the secondary system of the proposed PTM, increase slowly with $\mu$. Especially, the contribution of the varying $\mu$ to the amplitude of the upper boundary under 1:1/3 internal resonance condition is far more significant than those under $1: 1$ and $1: 1 / 1 / 2$ conditions. 

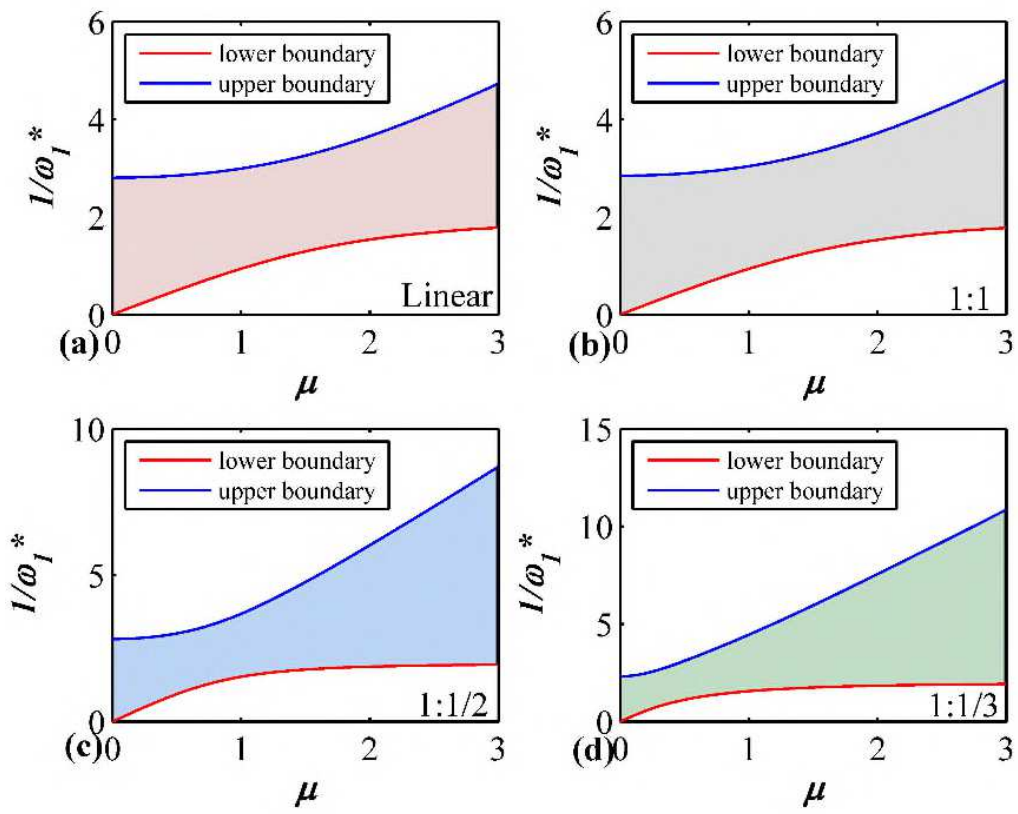

Fig. 5 The boundaries of PTM bandgap of versus $\mu$
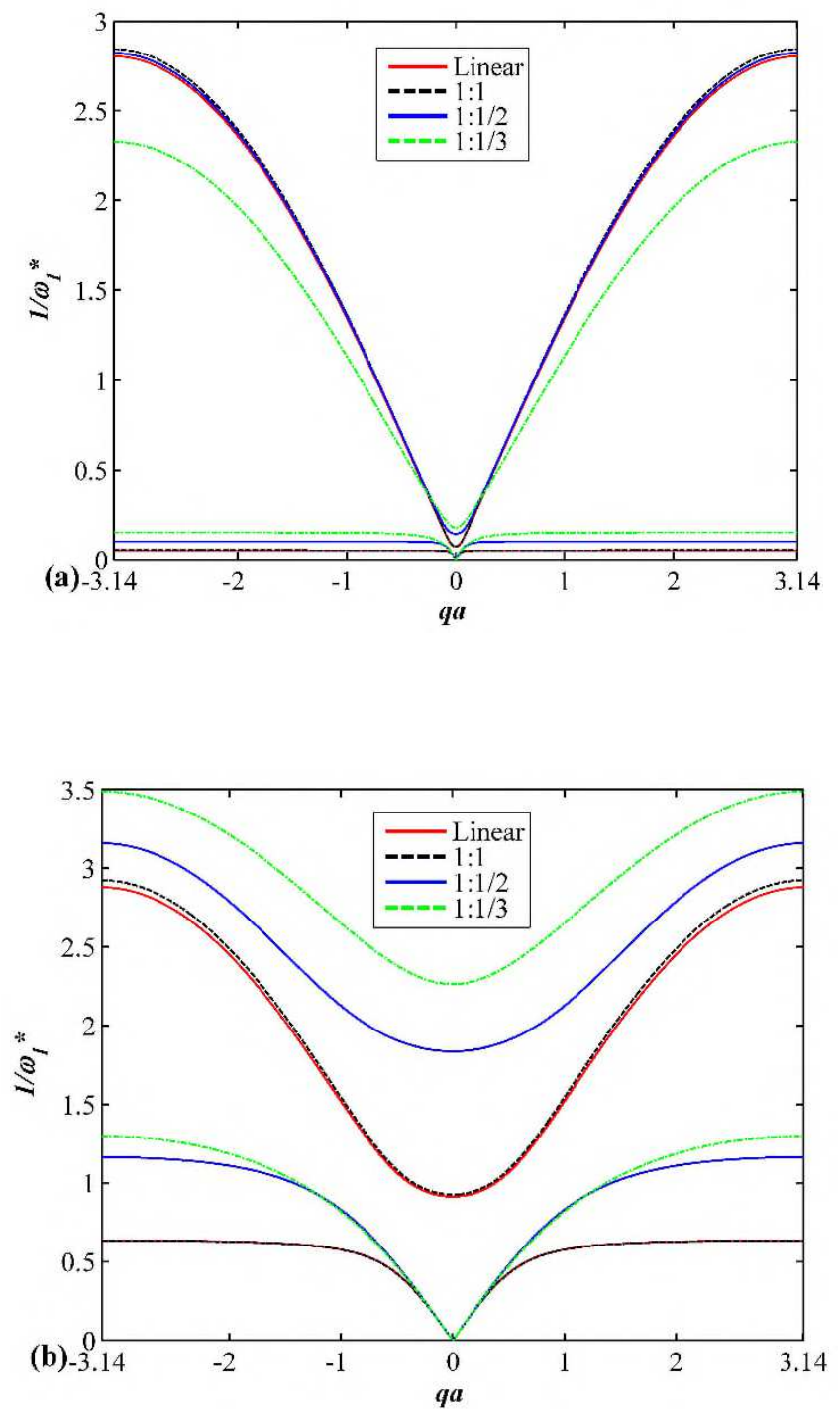


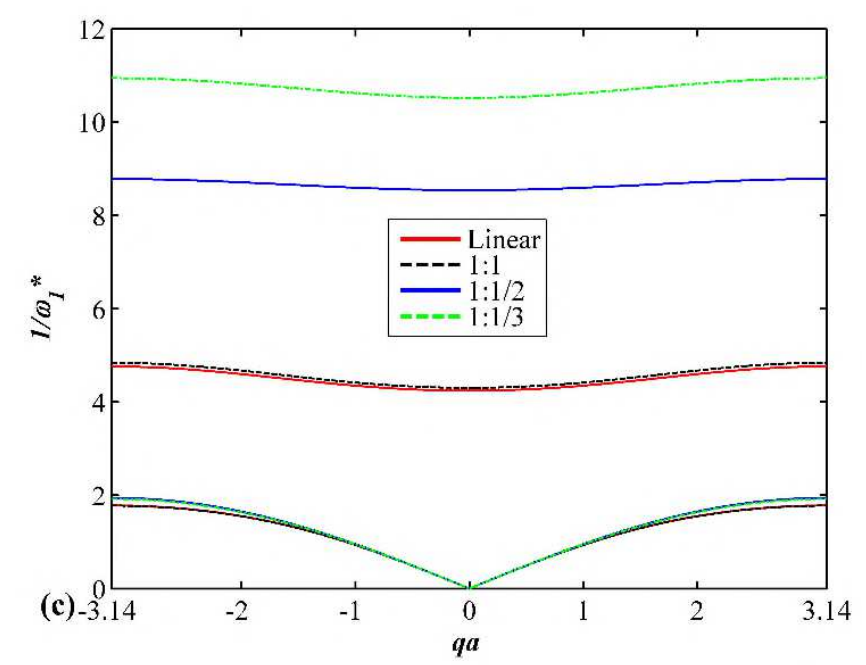

Fig. 6 The dimensionless dispersion curves of PTM under different cases:

$$
\text { (a) } \mu=0.005 \text {; (b) } \mu=0.65 \text {; (c) } \mu=3 \text {. }
$$

\subsection{The non-dimensional bandgap width of PTM versus different parameters}

With respect to the above analysis, Fig. 7 illustrates the non-dimensional width of the bandgap of PTM under different conditions versus $\mu$. It can be seen that the amplitude of non-dimensional width of the bandgap under 1:1, 1:1/2 and 1:1/3 internal resonance conditions are remarkable nonlinear curves because of the impact of nonlinear factors of pendulum. Under 1:1/3 internal resonance condition, the contribution of $\mu$ to the width of the bandgap is far more significant than those under other conditions, where PTM has the broadest bandgap when $\mu>0.75$. The curves under linear and 1:1 internal resonance condition are similar, but the amplitude of width of the bandgap caused by 1:1 internal resonance is always higher than those obtained from linearized dynamic model of PTM. It is indicated that the secondary system of PTM has a significant and nonlinear effect on the width of the bandgap, while the results under the linear condition only are incomplete and distorted against the actual situation. 


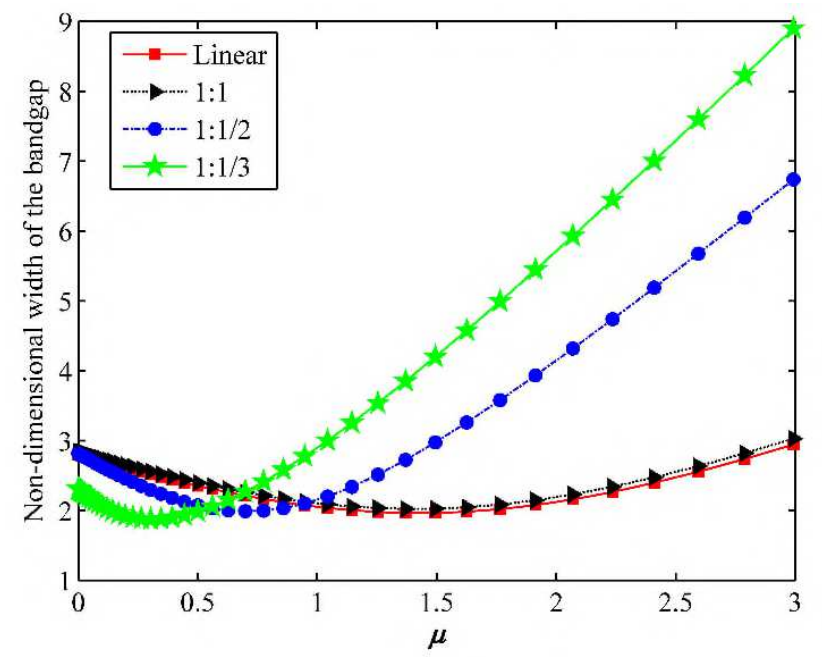

Fig. 7 The non-dimensional width of PTM bandgap versus $\mu$

In order to distinguish the influences of the dimensionless parameter $v$ on the nonlinear bandgap of PTM, Fig. 8 plots the non-dimensional width of the bandgap of PTM versus $v$ under different conditions. It is shown that the amplitude change of the bandgap width is in the form of high order curve, which increase nonlinearly with raised $v$. The upper boundary of the bandgap is more sensitive to the increasing value of the dimensionless parameter $v$, thus the overall width of bandgap is getting broader. Among the four curves, the amplitude curve caused by $1: 1 / 3$ internal resonance is always higher than the others when $v$ varies from 0.0016 to 0.003 , which implies that one can attain a wider stopband by the designing PTM under 1:1/3 internal resonance condition. 


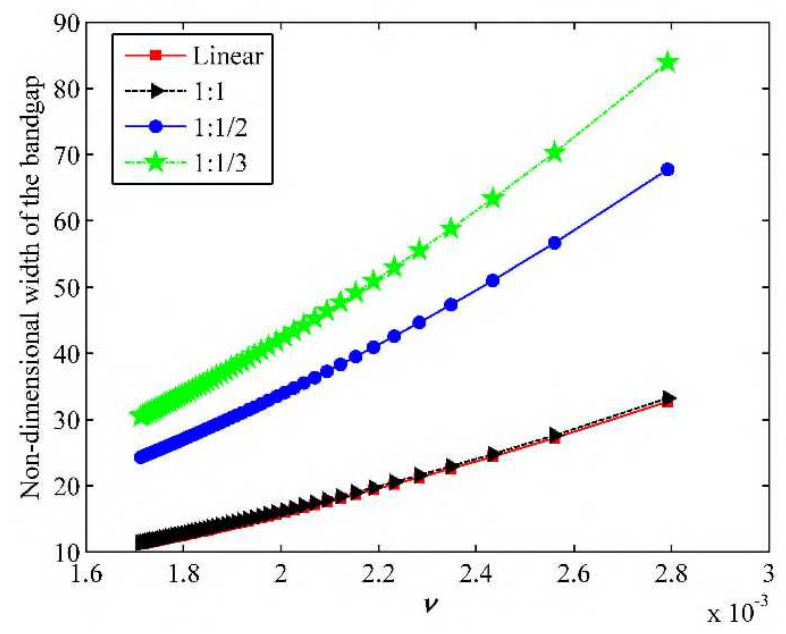

Fig. 8 The non-dimensional width of the bandgap of PTM versus $v$

According to the proposed nonlinear dynamic model of PTM, amplitude variation of width of the bandgap versus the mass ratio, $r$, is shown in Fig. 9. We can find that the amplitudes curve of all the four conditions have the similar increasing growth trend. As $r$ increases, the increasing rate of these curves will decrease slowly. Especially, it is noted that the bandgaps under linear and 1:1 internal resonance condition have the highest amplitude when $r<0.04$. The reason for this phenomenon is that when mass of the pendulum bob is small, the nonlinear effect of the pendulum bob motion is slight to be neglected while the primary system is the main factor that influence the width of the bandgap. As $r$ changes from 0.04 to 0.07 , the slops of the amplitude curves under 1:1/2 and 1:1/3 internal resonance conditions is larger than those of linearized model and 1:1 internal resonance conditions. And we can draw the conclusion that the mass ratio of pendulum bob and the primary mass has an important effect on the dynamic response of PTM, where we can enhance the width of the bandgap by increasing $r$ in a certain range. 


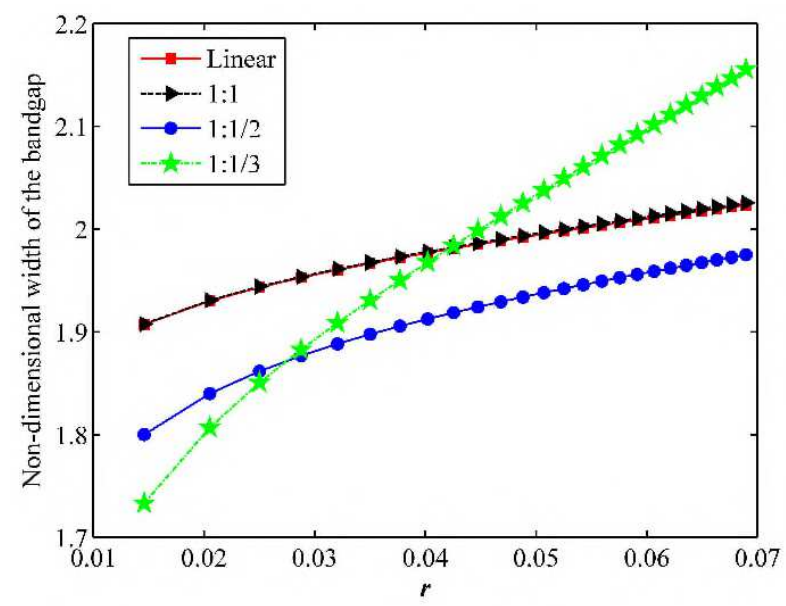

Fig. 9 The non-dimensional width of the bandgap of the PTM versus $r$

\section{Conclusions}

In this paper, a pendulum type metamaterial (PTM) is designed on the basis of the damping mechanism of the autoparametric vibration absorber. The dynamic model of the strongly nonlinear metamaterial is presented to investigate the dispersion characteristics. The theoretical equation of the nonlinear bandgap is proposed and compared to the results of linearized model. From the results presented in this paper, the following conclusions can be drawn.

1. With considering the nonlinear effects, the bandgaps under different internal resonances are substantially different from those under linear condition. The lower boundary of the bandgap is associated with the natural frequency of the primary system of the unit cell, and the secondary system has a strong effect on the upper boundaries. The nonlinear impact of the pendulum of PTM could enhance the width of the bandgap.

2. Due to the coupling effect of the torsion spring and the pendulum motion, the 
band gap of the proposed PTM possess obviously nonlinearity. Small value of dimensionless parameter, $\mu$, has a slight influence on the upper boundaries of the bandgaps. But the lower boundaries of the bandgap under various conditions is influenced by $\mu$ strongly when $\mu<1$.

3. The dimensionless parameter $v$, which is related to the characteristics frequency of the secondary system of PTM, has a remarkable and nonlinear effect on the width of the bandgap. The upper boundary of the bandgap is more sensitive to the increasing value of $v$.

4. When the mass coefficient, $r$, is slight, the nonlinear effect of the pendulum bob motion is slight due to the small mass of the pendulum bob. Meanwhile, the primary system is the main factor that affects the width of the bandgap. As $r$ increases, the width of the bandgap under 1:1/3 internal resonance rises significantly to higher than those under other conditions.

5. In this paper, the nonlinear analysis of dispersion characteristics of PTM is carried out under the harmonic loading. We also found that its capacity for vibration reduction could be more obvious when PTM is impulsed severely. And the stability of the pendulum motion might also have an impact on the bandgap structure. We will investigate the shock absorption characteristics and the stability of this metamaterial in our future researches.

\section{Appendix A. The dispersion equation of the Linearized dynamic model of PTM}

According to the traditional calculation method of metamaterial band gap, we 
make an attempt to linearize the dynamic model of the unit cell. When $\theta$ is small, the nonlinear terms in Eq. (8) can be eliminated. Then, the following equations of motion of the unit cell can be obtained

$$
\begin{gathered}
(M+m)+m l K_{1}\left(x_{L}-x_{R}\right)=f_{L} \\
m l^{2} K_{\theta} \theta+m l=0 \\
-K_{1}\left(x_{L}-x_{R}\right)=f_{R}
\end{gathered}
$$

The equation can be written as

$$
\bar{M}^{k} \bar{K}^{k}=\bar{f}
$$

where the matrixes $\bar{M}, \bar{x}, \bar{K}$ and $\bar{f}$ are given as

$$
\begin{array}{ccc}
\bar{M}=\left[\begin{array}{ccc}
M+m & m l & 0 \\
m l & m l^{2} & 0 \\
0 & 0 & 0
\end{array}\right], & \bar{x}=\left[\begin{array}{c}
x_{L}(t) \\
\theta(t) \\
x_{R}(t)
\end{array}\right] \\
\bar{K}=\left[\begin{array}{ccc}
K_{1} & 0 & -K_{1} \\
0 & K_{\theta} & 0 \\
-K_{1} & 0 & K_{1}
\end{array}\right], \quad \bar{f}=\left[\begin{array}{c}
f_{L}(t) \\
0 \\
f_{R}(t)
\end{array}\right]
\end{array}
$$

Considering the steady harmonic vibration of a periodic system, the solution of force and displacement can be set as

$$
\bar{x}=\left[\begin{array}{c}
x_{L}(t) \\
\theta(t) \\
x_{R}(t)
\end{array}\right]=\left[\begin{array}{c}
X_{L} \\
\theta \\
X_{R}
\end{array}\right] e^{i w t}, \quad \bar{f}=\left[\begin{array}{c}
f_{L}(t) \\
0 \\
f_{R}(t)
\end{array}\right]=\left\{\begin{array}{c}
F_{L} \\
0 \\
F_{R}
\end{array}\right\} e^{i w t}
$$

The following matrix equation can be obtained by using Bloch theorem

$$
\begin{aligned}
& \bar{x}=A \bar{X}=\left[\begin{array}{rr}
1 & 0 \\
0 & 1 \\
e^{-i q a} & 0
\end{array}\right]\left[\begin{array}{c}
X_{L} \\
\theta
\end{array}\right] \\
& \bar{f}=B \bar{F}=\left[\begin{array}{rr}
1 & 0 \\
0 & 1 \\
-e^{-i q a} & 0
\end{array}\right]\left[\begin{array}{c}
F_{L} \\
0
\end{array}\right] \\
& \bar{X}=\left[\begin{array}{c}
X_{L} \\
\theta
\end{array}\right], \quad \bar{F}=\left[\begin{array}{c}
F_{L} \\
0
\end{array}\right]
\end{aligned}
$$


where $A$ and $B$ are the matrixes as follows

$$
A=\left[\begin{array}{cc}
1 & 0 \\
0 & 1 \\
e^{-i q a} & 0
\end{array}\right], B=\left[\begin{array}{cc}
1 & 0 \\
0 & 1 \\
-e^{-i q a} & 0
\end{array}\right]
$$

And the Eq. (A.2) can be transformed to

$$
K A \bar{X}-\omega^{2} M A \bar{X}=B \bar{F}
$$

Then, multiply $A^{H}$ at the two sides of the equation at the same time, we have

$$
A^{H} K A \bar{X}-\omega^{2} A^{H} M A \bar{X}=A^{H} B \bar{F}
$$

where $A^{H}$, which is complex conjugate transpose matrix of $A$, is given as

$$
A^{H}=\left[\begin{array}{ccc}
1 & 0 & e^{i q a} \\
0 & 1 & 0
\end{array}\right]
$$

It is noted that $A^{H} B F_{r}$ is equal to zero vector. Therefore, Eq. (A.8) can be written in the form of the standard eigenvalue equation

$$
\left[K_{r}-\omega^{2} M_{r}\right] \bar{X}=0
$$

in which $K_{r}=A^{H} K A, M_{r}=A^{H} M A$.

Finally, by solving the eigenvalue equation, the following dispersion equation of the proposed PTM can be obtained as

$$
\omega=\sqrt{\frac{-\left(2 K_{1} m l^{2}(\cos (q a)-1)-K_{\theta}(M+m)\right)}{ \pm \sqrt{\left(\left(2 K_{1} m l^{2}(\cos (q a)-1)-K_{\theta}(M+m)\right)\right)^{2}-4\left(m M l^{2}\right)\left(2 K_{1} K_{\theta}(1-\cos (q a))\right)}}}
$$




\section{Appendix B. Nomenclature}

$a$

$f_{L}, f_{R} \quad$ The force exerted by the adjacent unit cells on the left and the right

$K_{1} \quad$ The linear spring of stiffness

$K_{\theta} \quad$ The torsional stiffness of the spring of secondary system of PTM

$L \quad$ The length of the simple pendulum

$M \quad$ The primary mass

$m \quad$ The mass of the pendulum bob

$P_{0} \quad$ The amplitude of the harmonic load

$q_{i}$

$q$

$Q_{i}$

$r$

$t$

$T$

$U$

$x$

$\&$

$\$$

$x_{L}, x_{R}$

$\theta$

$\theta$

The relatively generalized coordinate

The wave vector

The forces corresponding to generalized coordinates

The non-dimensional mass

The time

The kinetic energy of the unit cell

The potential energy of the unit cell

The displacement of $M$

The horizontal velocity of the primary mass

The acceleration of mass

The displacement of left end of the spring and the right end of the mass

The angle of rotation of the pendulum

The angular velocity of the pendulum bob 


$\begin{array}{ll}\Omega & \text { The angular acceleration of pendulum bob } \\ & \text { The angular velocity of the harmonic load } \\ \tau & \text { The non-dimensional displacement of the primary mass } \\ \tau & \text { The non-dimensional time } \\ \mu, v & \text { The non-dimensional parameters } \\ \omega_{1}, \omega_{2}, \omega_{3} & \text { The frequency of different part of the unit cell } \\ \omega_{1}{ }^{*}, \omega_{2}{ }^{*}, \omega_{3}{ }^{*} & \text { The non-dimensional frequency }\end{array}$

\section{Data Availability Statements}

The datasets generated during and/or analysed during the current study are available from the corresponding author on reasonable request.

\section{Declaration of Conflicting Interests}

The author(s) declared no potential conflicts of interest with respect to the research, authorship, and/or publication of this article.

\section{Acknowledgments}

The authors would like to gratefully acknowledge the support from National Natural Science Foundation of China (Grant No. 12072247). 


\section{References}

[1] Tan, K., Huang, H., Sun, C.: Optimizing the Band Gap of Effective Mass Negativity in Acoustic Metamaterials. Appl. Phys. Lett. 101, 241902 (2012)

[2] Finocchio, G., Casablanca, O., Ricciardi, G.: Seismic Metamaterials Based on Isochronous Mechanical Oscillators. Appl. Phys. Lett. 104(19), 509-379 (2014)

[3] Chen, Y., Wang, L.: Bio-Inspired Heterogeneous Composites for Broadband Vibration Mitigation. Sci. Rep. 17865(5), 1-11 (2015)

[4] Liu, Z., Zhang, X., Mao, Y.: Locally Resonant Sonic Materials. Science, 289(1), 1734-1736 (2000)

[5] Fang, N., Xi, D., Xu, J.: Ultrasonic Metamaterials With Negative Modulus. Nat. Mater. 5(6), $452-456(2006)$

[6] Hussein, M., Leamy, M., Ruzzene, M.: Dynamics of Phononic Materials and Structures: Historical Origins, Recent Progress, and Future Outlook. Appl. Mech. Rev. 66(4), 1-38 (2014)

[7] Jing, X., Meng, Y., Sun, X.: Soft Resonator of Omnidirectional Resonance for Acoustic Metamaterials with a Negative Bulk Modulus. Sci. Rep. 5(16), 1-8 (2015)

[8] Yasuda, H., Yang, J.: Reentrant Origami-Based Metamaterials with Negative Poisson's Ratio and Bistability. Phys. Rev. Lett. 114(18), 1-5 (2015)

[9] Tan, K., Huang, H., Sun, C.: Blast-wave Impact Mitigation Using Negative Effective Mass Density Concept of Elastic Metamaterials. Int. J. Impact Eng. 64(2), 20-29 (2014)

[10] Manimala, J., Huang, H., 2014: Dynamic Load Mitigation Using Negative Effective Mass Structures. Eng. Struct. 80(11), 458-468 (2014)

[11] Chen, Y., Barnhart, M., Chen, J.: Dissipative Elastic Metamaterials for Broadband Wave 
Mitigation at Subwavelength Scale. Compos. Struct. 136(2), 358-371 (2016)

[12] Huang, H., Sun, C.: Wave Attenuation Mechanism in an Acoustic Metamaterial with Negative Effective Mass Density. New J. Phys. 11(1), 1-15 (2009)

[13] Qureshi, A., Bing, L., Tan, K.: Numerical Investigation of Band Gaps in 3D Printed Cantilever-In-Mass Metamaterials. Sci. Rep. 6(28), 1-10 (2016)

[14] Yamamoto, T.: Acoustic Metamaterial Plate Embedded with Helmholtz Resonators for Extraordinary Sound Transmission Loss. J. Appl. Phys. 123(21), 1-9 (2018)

[15] Naify, C., Chang, C.: Membrane-Type Metamaterials: Transmission Loss of Multi-Celled Arrays. J. Appl. Phys. 109(10), 1-8 (2011)

[16] Zhang, H., Xiao. Y., Wen, X.: Flexural Wave Band Gaps in Metamaterial Beams with Membrane-Type Resonators: Theory and Experiment. J. Phys. D: Appl. Phys. 48(43), 1-11 (2015)

[17] Lott, M., Roux, P., Rupin, M.: Negative Index Metamaterial through Multi-Wave Interactions: Numerical Proof of the Concept of Low-Frequency Lamb-wave Multiplexing. Sci. Rep. 11(1), 1-8 (2021)

[18] Li, D., Hu, F., Zhang, H.: Identification of Early-stage Cervical Cancer Tissue Using Metamaterial Terahertz Biosensor with Two Resonant Absorption Frequencies. IEEE J. Sel. Top. Quant. 27(4), 8600107 (2021)

[19] Zhang, Y., Dong, H., Mou, N.: Tunable and Transparent Broadband Metamaterial Absorber with Water-Based Substrate for Optical Window applications. Nanoscale 13(78), 31-37 (2021) [20] Lin, Q., Zhou, J., Pan, H.: Numerical and Experimental Investigations on Tunable LowFrequency Locally Resonant Metamaterials. Acta Mech. Solida Sin. 10(2). 1-12 (2021) 
[21] Roberson, R.: Synthesis of a Nonlinear Dynamic Vibration Absorber. J. Franklin I. 254(3), 205-220 (1952)

[22] Yurchenko, D., Alevras, P., Zhou, S.: Nonlinear Vibration Mitigation of a Crane's Payload Using Pendulum Absorber. Mech. Syst. Signal Pr. 156(3), 107558 (2021)

[23] Alevras, P., Bobryk, R., Yurchenko, D. : Stability of an autoparametric pendulum system with impacts. . J. Sound and V. 333(26), 7233-7247 (2014)

[24] Benacchio, S., Malher, A.: Design of a Magnetic Vibration Absorber with Tunable Stiffnesses. Nonlinear Dynam. 85 (2), 893-911 (2016)

[25] Nishi, K., Nishiura, Y., Teramoto, T.: Reduction Approach to the Dynamics of Interacting Front Solutions in a Bistable Reaction-Diffusion System and its Application to Heterogeneous Media. Physica D. 398(3), 183-207 (2019)

[26] Hatwal, H., Mallik, A., Ghosh, A.: Non-Linear Vibrations of a Harmonically Excited Autoparametric System. J. Sound and V. 81(2), 153-164 (1982)

[27] Hatwal, H., Mallik, A, Ghosh, A.: Forced Nonlinear Oscillations of an Autoparametric System—Part 1: Periodic Responses. J. Appl. Mech. 50(3), 657-662 (1983)

[28] Hatwal, H., Mallik A, Ghosh, A.: Forced Nonlinear Oscillations of an Autoparametric System—Part 2: Chaotic Responses. J. Appl. Mech. 50(3), 663-668 (1983)

[29] Haxton, R., Barr, A.: The Autoparametric Vibration Absorber. J. Eng. Indust. 94(1), 119$125(1972)$

[30] Emrah, G., Atila, E.: Analysis of Free Pendulum Vibration Absorber Using Flexible MultiBody Dynamics. Shock Vib. 2016, 1-19 (2016)

[31] Oueini, S., Chin, C., Nayfeh, A.: Dynamics of a Cubic Nonlinear Vibration Absorber. 
Nonlinear Dynam. 20(3), 283-295 (1999)

[32] Pai, P., Rommel, B.: Non-Linear Vibration Absorbers Using Higher Order Internal Resonances. J Sound Vib. 234(5), 799-817 (2000)

[33] Ji, J.: Design of a Nonlinear Vibration Absorber Using Three-to-One Internal Resonances. Mech. Syst. Signal Pr. 42(1-2), 236-246 (2014)

[34] Mahmoudkhani, S.: Dynamics of a Mass-Spring-Beam with 0:1:1 Internal Resonance Using the Analytical and Continuation Method. International J. Nonlin. Mech. 97(12), 48-67 (2017)

[35] Fronk, M., Leamy, M.: Higher-Order Dispersion, Stability, and Waveform Invariance in Nonlinear Monoatomic and Diatomic Systems. J. Vib. Acoust. 139(5), 051003 (2017)

[36] Manktelow, K., Narisetti, R., Leamy, M.: Finite-Element Based Perturbation Analysis of Wave Propagation in Nonlinear Periodic Structures. Mech. Syst. Signal Pr. 39(1), 32-46 (2013) [37] Bilal, O., Foehr, A., Daraio, C.: Bistable Metamaterial for Switching and Cascading Elastic Vibrations. P. Nati. A. Sci. 114(18), 4603-4606 (2017)

[38] Fang, X., Wen, J., Benisty, H.: Ultrabroad Acoustical Limiting in Nonlinear Metamaterials due to Adaptive-Broadening Band-Gap Effect. Phys. Rev. B. 101(10), 1-10 (2020)

[39] Sheng, P., Fang X.: Vibration Properties and Optimized Design of a Nonlinear Acoustic Metamaterial Beam. J. Sound Vib. 492(11), 1-17 (2021)

[40] Brooke, D., Liclaire, P.: Acoustic Metamaterial for Low Frequency Sound Absorption in Linear and Nonlinear Regimes. J. Sound Vib. 485(11), 1-20 (2020) 